\title{
Assessing the risk of minimally-invasive surgery: a metrological approach
}

\author{
Maria Nau ${ }^{\mathrm{a}}$, Sebastian Pollmanns and Robert Schmitt \\ Chair for Metrology and Quality Management, WZL of RWTH Aachen University, \\ Steinbachstr. 19, 52074 Aachen
}

\begin{abstract}
Principles of uncertainty evaluation for measurement processes and capability studies for production processes are established in many producing enterprises to ensure capable processes. Together with further quality management methods this allows to estimate and minimize the risk of producing defective parts and of taking wrong decisions about the part's conformity with its specification. This understanding is also desirable for a completely different field, for medical surgery. To quantify the risk of minimally invasive surgery for an individual patient, principles of metrology and production engineering are transferred to the medical domain and are exemplarily applied to minimally invasive surgery at the inner ear. It is shown how the main influencing factors, the uncertainty of the imaging and image processing and the uncertainty of the navigation process of the surgical instrument can be estimated with methods from production metrology. Furthermore, it is shown how these factors contribute to quantifying the patient's risk of a specific surgery by contributing to a therapeutic risk indicator (TRI).
\end{abstract}

\begin{abstract}
Les principes et méthodes pour estimer l'incertitude de mesure et l'aptitude de procès de manufacture ont été établis dans beaucoup d'entreprises pour obtenir des procès capables. En combinaison avec d'autres méthodes de gestion de qualité, le risque de fabriquer des pièces endommagées et de prendre une décision non-fondée concernant la qualité d'une pièce peut être réduit. Ce concept est aussi souhaitable pour un autre domaine : les opérations chirurgicales mini-invasives. Les principes de métrologie et de technique de production sont transférés dans le domaine médical pour quantifier le risque d'une opération mini-invasive pour un patient, par exemple d'une opération mini-invasive de l'oreille interne. Ce travail montre comment les facteurs principaux, l'incertitude d'imagerie et l'incertitude de naviguer les instruments chirurgicaux, peuvent être estimés avec les principes de métrologie manufactures. En plus, cela montre comment ces facteurs contribuent à quantifier le risque d'une opération mini-invasive et à l'index thérapeutique de risque (TRI).
\end{abstract}

\section{Introduction}

Principles of uncertainty evaluation for measurement processes [1] and capability studies for production processes [2] have been known for several years and are established in many producing enterprises where they ensure capable and controlled processes. The risk of producing defective parts and of taking wrong decisions about a part's conformity with its specification is generally known and can be minimized by applying quality management methods such as a failure mode and effect analysis and statistical process control. The ability to quantify the processes' risk is also desirable for a

\footnotetext{
a Corresponding author: m.nau@wzl.rwth-aachen.de
}

completely different field, for medical surgery. Especially for new and minimally invasive surgery it is very important to estimate and state the risk of the surgical intervention for the individual patient.

Minimally invasive surgery has been established in different fields of medicine and has sometimes even become a standard procedure such as laparoscopy. In otolaryngology approaches to e.g. insertion of cochlear implants by a minimally invasive procedure have been reported by several research groups [3-5]. Other possible applications are the treatment of acoustic neuromae and the recovery of tissue samples [6]. Minimally-invasive scull surgery is usually image-guided as the affected 
cranial area includes several sensitive structures such as nerves and blood vessels. For describing the quality of these image-guided systems the term "accuracy" is often used in medicine. Authors describe for example mechanical and application accuracies [7] and overall accuracy [8] and calculate performance indicators such as the fiducial registration error (FRE) and the target registration error (TRE). Although there is an increasing demand for evidence based medicine $[9,10]$ there are no binding guidelines and standards about how to estimate the uncertainty of surgical processes and the measurement uncertainty of e.g. the medical computed tomography $(\mathrm{CT})$.

\section{The medical scenario transferred to production metrology}

The mentioned possible applications for minimally invasive surgery at the inner ear all imply that boreholes in the scull are needed to reach the target area (Figure 1). Therefore, it is necessary to navigate a drill along a planned trajectory to ensure that nearby vital structures like nerves, blood vessels or brain tissue will not be damaged.

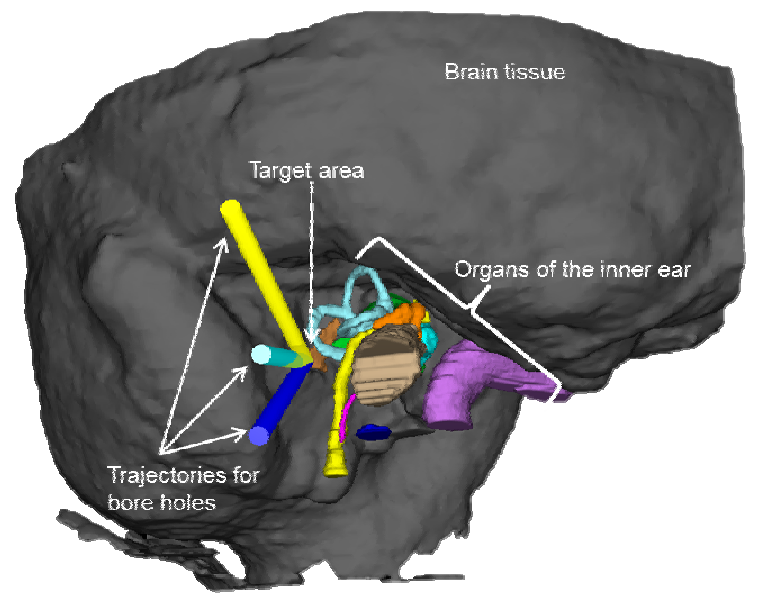

Figure 1. Planned trajectories for the bore holes with surrounding sensitive structures (organs of the inner ear with nerves and blood vessels and brain tissue).

As defined in a previous work, the main factors that influence the quality of this procedure is the uncertainty of imaging and image processing $u_{\text {imag }}$ (in this case computed tomography) on the one hand and the uncertainty of positioning the drill $u_{\text {navi }}$ on the other hand [11]. Taking this into account a therapeutic risk indicator (TRI) based on the calculation of the process capability index $c_{\mathrm{pk}}$ [2] and on ISO 14253-1 [12] of production metrology has been proposed [13] (1).

$$
\begin{gathered}
\mathrm{TRI}=\min \left(\left[\left(\mathrm{USL}_{\mathrm{S}}-\mathrm{U}_{\text {imag }}\right)-\left(\mathrm{x}_{0}+\mathrm{r}\right)\right] / \mathrm{U}_{\text {navi }} ;\right. \\
\left.\left[\left(\mathrm{x}_{0}+\mathrm{r}\right)-\left(\mathrm{LSL}-\mathrm{U}_{\text {imag }}\right)\right] / \mathrm{U}_{\text {navi }}\right)
\end{gathered}
$$

Transferring the medical scenario to production metrology, the uncertainty due to the imaging and image processing corresponds to the measurement uncertainty which has to be considered at the specification limits according to ISO 14253-1 (Figure 2). The navigation of the drill to set the boreholes corresponds to the production process.

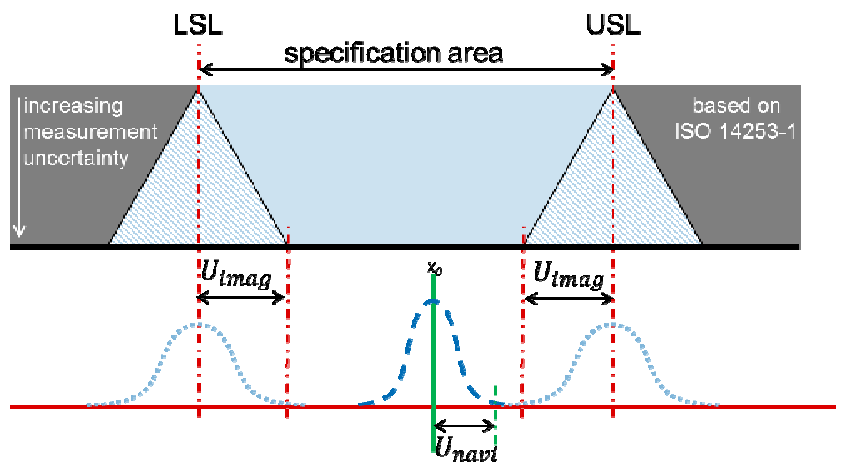

Figure 2. Upper and lower specification level (USL, LSL) are reduced by the extended uncertainty due to imaging process and image processing $U_{\text {imag }}$ according to ISO 14253-1. The centricity $\mathrm{x}_{0}$ and the extended uncertainty $\mathrm{U}_{\text {navi }}$ of the instrument navigation have to lie in the reduced specification area to ensure a capable process.

The tolerance zone between the upper and lower specification limit in the medical scenario is defined by the planned trajectory and its distance to the closest vital structures. The automatic planning of the trajectories is based on a software tool introduced in [5].

\section{Quantifying the uncertainties that influence the therapeutic risk measure}

In order to calculate the TRI, the distance of the trajectory to the sensitive structures, as well as the uncertainties of the imaging and navigation processes must be estimated.

\subsection{Realizing the navigation process and estimating its uncertainty}

\subsubsection{A mechanical positioning system for the drill}

To position the drill according to the planning of the surgery, reference structures are defined that can be detected in the CT image data as well as on the patient during surgery.

Several positioning devices for head surgery can be found in the literature (e.g. [3, 14, 15]) but few are available commercially. The ones that are available are either very large like the stereotactic frame or they are individual for each patient $[16,17]$. Due to this a prototype has been developed in this project for positioning a surgical instrument to show how the uncertainty of the navigation process can be estimated based on principles of production metrology.

The proposed positioning device (Figure 3) consists of a stable platform which is suitable for CT-scan and that can be attached to the scull of the patient by surgical screws. Reference structures made of ruby balls are fixed to the platform. To position the surgical instrument correctly two linear ( $\mathrm{x}$ - and y-axis) as well as two rotatory axes can be attached to the platform. 


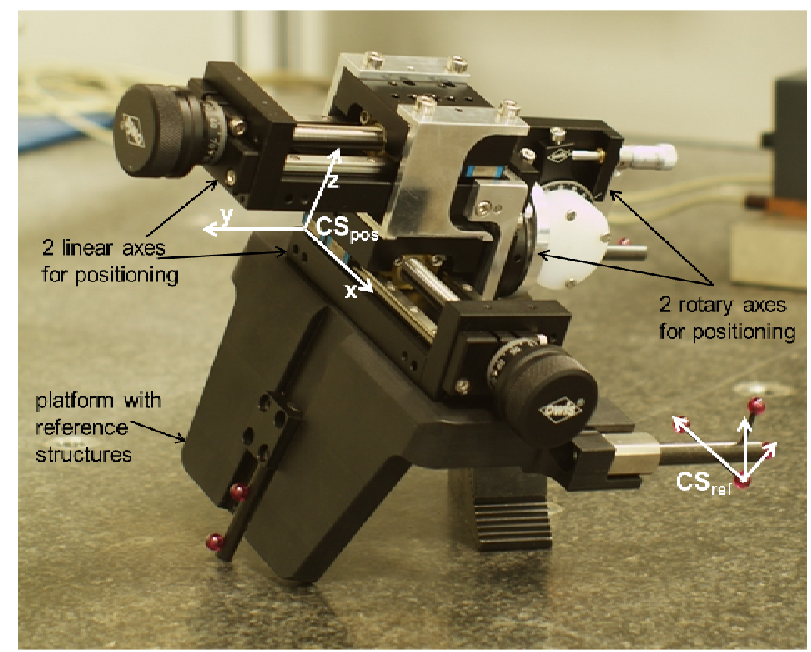

Figure 3. Prototype of a device to position instruments for minimally invasive surgery at the scull base consisting of a platform with reference structures (ruby balls) and 2 linear and 2 rotary axes which are used to define 2 local coordinate systems $\mathrm{CS}_{\text {ref }}$ and $\mathrm{CS}_{\mathrm{pos}}$

The parameters for the 4 axes can be calculated based on the planned trajectories of the software tool [5] with the ruby balls as reference structures in the image data as well as on the patient: A local Cartesian coordinate system $C S_{\text {ref }}$ can be defined using the reference structures. Another local Cartesian coordinate system $C S_{\text {pos }}$ is defined using characteristic points on the guide rail of the lower linear axis (x-axis). The parameters of the trajectories are calculated in the planning tool based on the image data and can be defined in the $C S_{\text {ref. }}$ The transformation $C S_{r e f} \rightarrow C S_{p o s}$ is known with an uncertainty due to the mechanical interface between platform and positioning system which can be estimated.

\subsubsection{Estimating the uncertainty of the mechanical positioning system}

The uncertainty of the navigation process depends on the design of the positioning system. In the case of the proposed positioning device, the following aspects influence the uncertainty of the navigation process $u_{\text {navi }}$ :

the uncertainty of the linear axes $u_{\text {lin }}$

the uncertainty of the rotary axes $u_{\text {rot }}$

the calibration uncertainty $u_{c a l}$

Furthermore, the uncertainty due to the mechanical interface between platform and positioning unit $u_{m o n}$ is important. These contributions to the uncertainty of the calibration process can be estimated experimentally and this work focuses on the uncertainty of the linear axes $u_{\text {lin. }}$.

For the experiments a calibrated double-ball-bar is fixed in the tool centre point of the positioning unit (Figure 4) and the whole unit is setup on a coordinate measuring machine (CMM) (Prismo Vast, Zeiss).

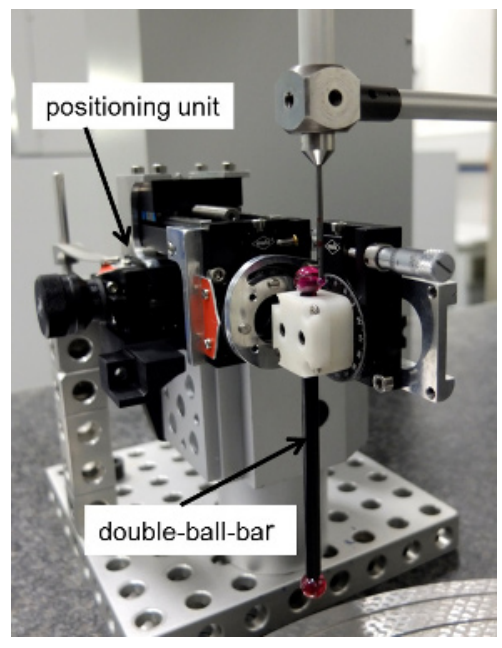

Figure 4. Test setup with the positioning unit and the doubleball-bar on the CMM

For estimating the uncertainty of the linear axes the following positions are approached 10 times each for the $\mathrm{x}-$ and $\mathrm{y}$-axes of the positioning unit and for all combinations of these positions (compare Figure5): $0 \mathrm{~mm}, 11,25 \mathrm{~mm}, 25,12 \mathrm{~mm}, 43,8 \mathrm{~mm}$. The position of the upper ball's centre of the calibrated double-ball-bar is measured each time to evaluate the effect of the axes' uncertainty in the tool centre point.

The uncertainty $u_{3}$ (in $\mu \mathrm{m}$ ) of the CMM that is used for the experiments is defined as (2) with $l$ in $\mathrm{mm}$.

$$
\mathrm{u}_{3}=2,5+1 / 300
$$

The standard deviations of the positions are with one exception smaller than $1,5 \mu \mathrm{m}$ in $x$ as well as in $y$ direction. As the uncertainty of the CMM for the measuring volume of $50 \mathrm{~mm}$ is $2,67 \mu \mathrm{m}$ and therewith larger than the calculated standard deviation, no conclusion can be drawn regarding the positioning uncertainty.

The systematic (mean) positioning error of the $\mathrm{x}$-axis increases with the increasing position of the $y$-axis (Figure 5). This shows that the axes are not exactly perpendicular to each other. This systematic error is about factor 100 of the standard deviation but can be compensated e.g. based on the geometric error compensation for machines [18].

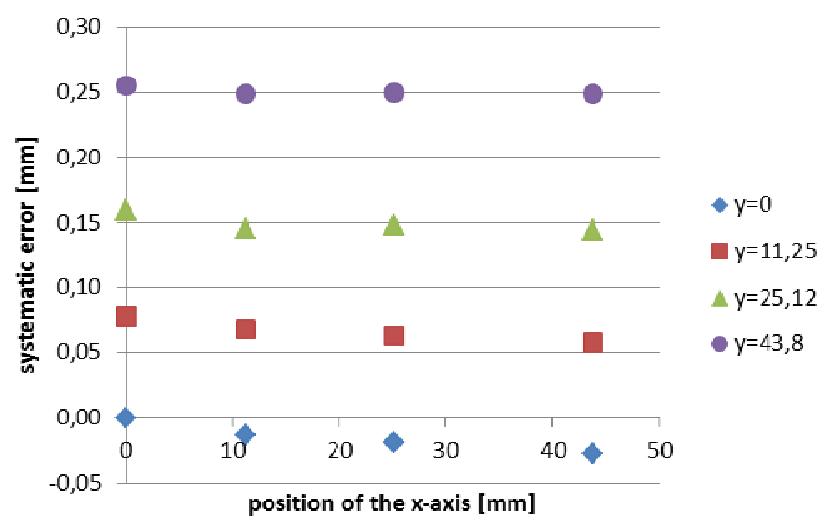

Figure 5. Systematic error of positioning the $x$-axis depending on the position of the y-axis 


\subsection{Estimating the uncertainty of the imaging process}

There are three methods for estimating the uncertainty of the CT imaging [19]: to estimate the uncertainty budget based on ISO 98-3[1], to use simulation based on ISO 983 Supplement 1 [20] or to use a calibrated workpiece based on ISO 15530-3 [21].

To estimate the uncertainty of CT imaging and image processing for the described application of minimally invasive surgery at the inner ear a test specimen (Figure 6 ) is developed which provides the anatomical structures of the region of interest as well as calibrated structures [13]. This allows to estimate the systematic deviation as well as the standard deviation. These anatomical and calibrated structures are embedded in a medical phantom made of CT equivalent material which imitates the behaviour of skin, brain tissue and the cranial bone regarding $\mathrm{CT}$ imaging.

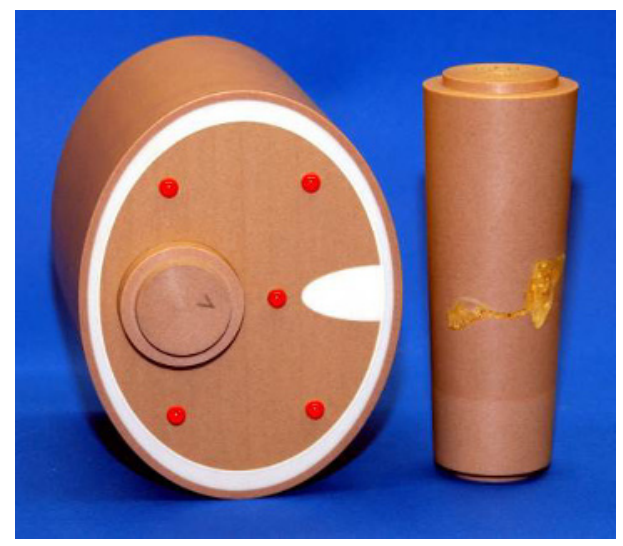

Figure 6. Test specimen with anatomical as well as calibrated structures embedded in CT-equivalent material

\section{Conclusion and Outlook}

It is a promising approach to the transfer of methods for process characterization and uncertainty estimation from production metrology to the medical domain as these methods have been applied for decades in production engineering and are therefore well known and approved in that field. It has been shown how the navigation of surgical instruments (e.g. drilling instruments) can be realized using mechanical positioning devices and reference structures and how the uncertainty of this process can be estimated experimentally.

In an earlier study [13] the distance from a planned centre of the trajectories to the closest sensitive structure has been determined for six patients and two different target areas (round window and petrous apex) being $2 \mathrm{~mm}$ maximally over these patients for the petrous apex as target area. The data which corresponds to a one-sided tolerance shows that the area that can be used for the boreholes is very tight. Therefore, it is desirable to decrease all uncertainties on the process as much as possible to decrease the risk of the surgery for the patient. Therefore, e.g. the systematic error of the linear axes shown in Figure 4 has to be compensated.
In future studies it will be necessary to investigate the influence of other mentioned factors like the uncertainty of the rotary axes. Furthermore, it will be possible to define the uncertainty of the $\mathrm{CT}$ imaging for a specific measurement task with the proposed test specimen (Figure 6). Like this it will be possible to calculate the TRI and the patient's risk for minimally invasive surgery.

\section{Acknowledgement}

We would like to thank the German Research Foundation DFG for the support and the funding of the depicted research within the research group MUKNO (FOR 1585). We would also like to thank our project partners within this research group, the Heinrich Heine University of Düsseldorf with Prof. J. Schipper, head of the research group, Dr. S. Hansen and Dr. P. Becker as well as the Technical University of Darmstadt with M. Becker.

\section{References}

1. International Organization for Standardization, Guide to the expression of uncertainty measurement (GUM: 1995, 1st edn.) (2008)

2. International Organization for Standardization, Statistical methods in process management Capability and performance - Part 1: General principles and concepts (2009)

3. L.B. Kratchman, Blachon Grégoire S., T.J. Withrow, R. Balachandran, R.F. Labadie, R.J. Webster, Robotics science and systems (2010)

4. A. Hussong, T.S. Rau, H. Eilers, S. Baron, B. Heimann, M. Leinung, T. Lenarz, O. Majdani, $E M B C$, ed. by IEEE, 5593 (2008)

5. M. Becker, R. Gutbell, I. Stenin, S. Wesarg, Clinical Image-based Procedures, 59 (2013)

6. I. Stenin, S. Hansen, M. Becker, J. Hirschfeld, B. Bojovic, T. Klenzer, J. Schipper, CURAC2012@MEDICA, CD V 12 (2012)

7. R.L. Galloway, R.J. Maciunas, Crit Rev Biomed Eng 18, 181 (1990)

8. R. Steinmeier, J. Rachinger, M. Kaus, O. Ganslandt, W. Huk, R. Fahlbusch, Stereot Funct Neuros 75, 188 (2000)

9. G.H. Guyatt, A.D. Oxman, G.E. Vist, R. Kunz, Y. Falck-Ytter, H. Schunemann, Brit Med J 336, $995-$ 999B (2008)

10. D.L. Sackett, W.M. Rosenberg, J Public Health Med 17, 330 (1995)

11. R. Schmitt, M. Nau, S. Pollmanns, S. Hansen, J. Schipper, CURAC2012@MEDICA, CD II 11 (2012)

12. International Organization for Standardization, Geometrical Product Specifications (GPS) Inspection by measurement of workpieces and measuring equipment - Part 1: Decision rules for proving conformity or nonconformity with specifications (1999)

13. M. Nau, S. Pollmanns, R. Schmitt, 11th ISMTII, CD $07 \quad 14$ (2013) 
14. R.F. Labadie, J. Mitchell, R. Balachandran, J.M. Fitzpatrick, Int J Comput Ass Rad 4, 273 (2009)

15. M.-L. Lin, B.-D. Yang, Y.-H. Wang, C.-L. Yang, J.-L. Wang, Int J Med Robot Comp, 7, 423 (2011)

16. J.M. Fitzpatrick, P.E. Konrad, C. Nickele, E. Centinkaya, C. Kao, Stereot Funct Neuros, 25 (2005)

17. P.E. Konrad, J.S. Neimat, H. Yu, C.C. Kao, M.S. Remple, P.-F. D'Haese, B.M. Dawant, Stereot Funct Neuros, 89, 34 (2011)

18. H. Schwenke, W. Knapp, H. Haitjema, A. Weckenmann, R. Schmitt, F. Delbressine, CIRP Ann 57, 660 (2008)

19. Verein deutscher Ingenieure, Computertomografie in der dimensionellen Messtechnik - Bestimmung der Messunsicherheit und der Prüfprozesseignung von Koordinatenmessgeräten mit CT-Sensor (2013)

20. International Organization for Standardization, Uncertainty of measurement - Part 3: Guide to the expression of uncertainty in measurement (GUM:1995) - Supplement 1: Propagation of distributions using a Monte Carlo method, (2009)

21. International Organization for Standardization, Geometrical product specifications (GPS) Coordinate measuring machines (CMM): Technique for determining the uncertainty of measurement - Part 3: Use of calibrated workpieces or measurement standards (2012) 